\title{
A Case Report of Middle Aortic Syndrome: A Rare Vascular Disorder
}

\author{
NC Mandal, MF Hossain, AA Mamun, NK Dey, MN Sabah, MA Arif, S Samad, MR Rahman, AHM \\ Bashar, GMM Hossain, E Hakim, SAN Alam, MK Hasan, QA Azad \\ Department of Vascular Surgery, NICVD, Dhaka.
}

\begin{abstract}
Keywords: $\quad$ The middle aortic syndrome (MAS) is rare (about $0.5-2 \%$ of all the cases of aortic coarctation) vascular Middle aortic syndrome, Abdominal aortic coarctation,

Abstract: disorder characterized by severe narrowing in the descending thoracic aorta, abdominal aorta, or both. It can be congenital or acquired due to several conditions.MAS may present clinically as uncontrolled hypertension, abdominal angina or lower limb claudication. Surgical treatment is effective in controlling symptom and improves life expectancy.
\end{abstract} Hypertension, Lower limb claudication.

(Cardiovasc. j. 2013; 6(1): 60-62)

\section{Introduction:}

Middle aortic syndrome (MAS) is a rare entity affecting children and young adults, predominantly females. The middle portion of the vessel (between the arch and bifurcation) is usually involved and named as "middle aortic syndrome". ${ }^{1}$

The etiology of the syndrome is controversial. Some authors report a congenital origin of the disease due to failure of fusion of the paired dorsal aortae during the fourth week of gestation, ${ }^{2,3}$ whereas others believe it is the healing phase of aortitis. ${ }^{4,5}$

Middle aortic syndrome can present as hypertension or lower limb claudication or abdominal angina. MAS is characterized radiologically by severe narrowing of abdominal aorta and its branches. Most of the patients usually die due to progressive severe hypertension before the age of 35-40 yrs if left untreated. ${ }^{1,6}$ Surgical treatment is challenging and requires careful assessment and planning for corrective surgery.

\section{Case Report:}

A 22 years old hypertensive, nondiabetic, nonsmoker, unmarried girl presented to the department of vascular surgery of NICVD with intermittent claudication of both lower limbs for 5 years. The claudication distance was declined to few feet only over the last 5 years. Both her femoral pulses were of small volume and distal pulses in either of the lower limbs were not palpable. All the upper limb pulses were palpable. ABPI was 0.625 on right and 0.58 on left side. She gave history of episodes of painful swelling of large joints of upper and lower limbs. Her blood pressure was 160/90 mm Hg in upper limbs and 90/50mm $\mathrm{Hg}$ in lower limbs and was on irregular antihypertensive medication. She was taking Indomethacin and Methotraxate for joint pain. Her menstrual history was normal and nothing was suggestive of tuberculosis in the past.

Hemogram was normal except ESR was $95 \mathrm{~mm}$. Renal \& liver function, serum electrolytes, lipid-profile were normal. Chest X-ray, ECG and Echocardiogram were also normal. Serology revealed high CRP $(48 \mathrm{mg} / \mathrm{L})$ and positive ANA, whereas C-ANCA, P-ANCA \& Anti-phospholipid antibodies were negative. A high resolution computer tomography angiogram showed severe tubular narrowing of distal abdominal aorta.

Address Correspondence: Dr. Naresh Chandra Mandal, Department of Vascular Surgery, National Institute of Cardiovascular Diseases, Dhaka, Bangladesh. E-mail: dr.ncm62@gmail.com. 


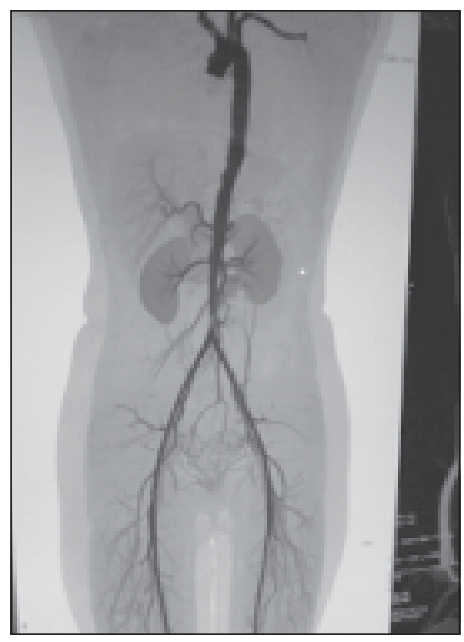

Fig.-1: CT angiogram showing Infra Renal Abdominal Aortic Stenosis.

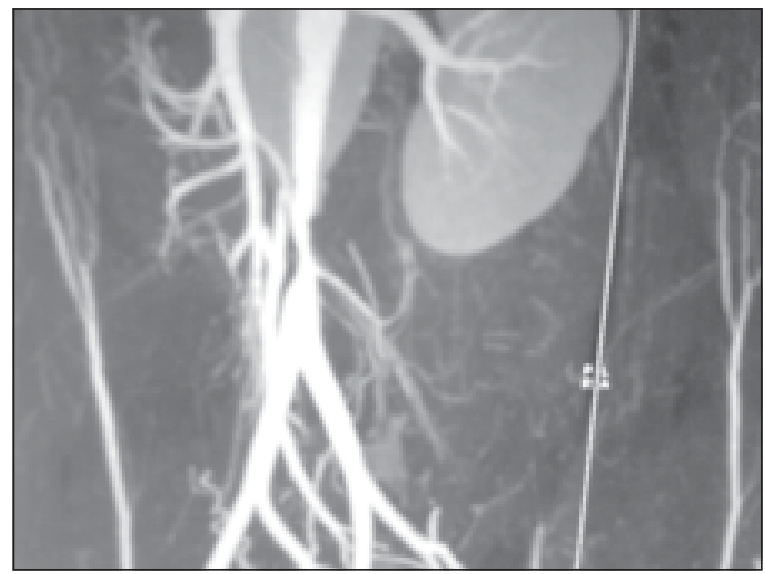

Fig.-2: CT angiogram showing Infra Renal Abdominal Aortic Stenosis.

Based on clinical picture, serological and CT angiogram findings, the diagnosis of middle aortic syndrome associated with Takayasu's arteritis was made and corrective surgery was planned. The lesion involved the infrarenal abdominal aorta extending from about $5 \mathrm{~cm}$ distal to the origin of the left renal artery up to about $2.5 \mathrm{~cm}$ above the aortic bifurcation. After preoperative optimization, laparotomy was done through midline incision under thoracic epidural anesthesia. Proximal control of abdominal aorta just distal to the renal arteries was made transperitonialy \& vascular clamp was applied proximal and distal to the stenotic region of the abdominal aorta. Aortotomy was done longitudinally at the stenotic segment and patch reconstruction was done with Dacron patch (size of patch was $2.5 \mathrm{~cm} \times 5 \mathrm{~cm}$ ). Perioperative period was uneventful. She was discharged on $12^{\text {th }}$ post operative day with a blood pressure of $120 / 70 \mathrm{~mm}$ $\mathrm{Hg}$ in the upper limbs and $110 / 65 \mathrm{~mm} \mathrm{Hg}$ in the lower limbs with captropil $25 \mathrm{mg} /$ day. There was no lower limb claudication or abdominal angina. Peripheral pulses were palpable and ABPI was 0.9 bilaterally.

\section{Discussion:}

Coarctation of aorta is usually located in the thoracic aorta just distal to or sometime even proximal to ligament arteriosum. Middle aortic syndrome (MAS) is a rare entity characterized by localized narrowing of abdominal or distal thoracic aorta. ${ }^{1,7}$ It constitutes about $0.5-2 \%$ of all the cases of aortic coarctation. ${ }^{7}$ MAS was first described by Sen et al, in $1963 .{ }^{8}$ Male to female ratio is $3: 1 .{ }^{1}$ Although the cause of MAS is not well known, it may be congenital or due to inflamatory, developmental disorder and infection. Congenital coarctation has been thought to be due to incomplete fusion or overfusion of embryonic dorsal aortas during 4th week of gestation. ${ }^{9,10}$ Another hypothesis implicates intra-uterine injury or infection, particularly rubella is the risk factor that precipitates aortic hypoplasia. ${ }^{7}$ Acquired MAS is associated with neurofibromatosis, William's syndrome, Alagille syndrome, fibromuscular dysplasia, retroperitoneal fibrosis (Ormond disease), mucopolysaccharidosis, foetal alcohol syndrome and giant cell arteritides including temporal (cranial) and Takayasu arteritis. ${ }^{1,11}$

It usually presents as hypertension in young age group which is typically unresponsive to medical therapy. It may also present as lower limb claudication or abdominal angina. The life expectancy of patients with untreated MAS is 30-40 years. The main reason of death is cardiovascular complications of progressive hypertension including cerebrovascular accidents, cardiomegaly, left heart failure and coronary artery disease. ${ }^{1,6}$

The severity of hypertension is the primary indication for intervention and the factor determining procedural timing. Endovascular therapy may provide a sound minimally invasive treatment in MAS caused by discrete aortic stenosis that do not encompass the mesenteric and renal arteries. ${ }^{1}$ 
Open surgery is the primary treatment of tubular aortic narrowing associated with renovascular hypertension and visceral artery stenosis. For patients with active vasculitides surgery is not recommended in active phase of disease. ${ }^{1,6,7}$ For children best results are achieved if definitive treatment can be delayed till they have achieved full growth. ${ }^{1}$

Aim of surgery is to control hypertension by improving tissue perfusion to renal, mesenteric vessels and lower limbs. Indications of surgery therefore depend upon severity of hypertension, lower limb claudication, involvement of kidneys and mesenteric ischaemia. ${ }^{1,7}$ Surgical repair depends upon anatomy of the lesion and involvement of visceral arteries. Options include aorto-aortic bypass, patch aortoplasty, renal and mesenteric arterial reconstruction. ${ }^{7}$ Results of surgery are good in most cases with improvement or normalization of blood pressure in $70 \%$ cases. $^{1}$

In our case we performed a patch aorto plasty of stenotic segment of the abdominal aorta. Both unilateral and bilateral renal artery revascularizations have been documented in literature. ${ }^{12}$

\section{Conclusion:}

Middle aortic syndrome is a rare cause of uncontrolled hypertension with poor outcome if left untreated. Surgical correction is both feasible and effective in middle aortic syndrome. There is a need to promptly and correctly diagnose uncontrolled hypertension in young adults. Timely referral to a vascular surgeon for corrective surgery will reduce complications and mortality.

\section{References:}

1. Delis KT, Gloviczki P. Middle aortic syndrome: from presentation to contemporary open surgical and endovascular treatment. Perspect Vasc Surg Endovasc Ther 2005; 17: 187-203

2. Graham LM, Zelenock GB, Erlandson EE. Abdominal aortic coarctation and segmental hypoplasia. Surgery 1979; 86: 519-529.

3. Maycock W. Congenital stenosis of the abdominal aorta. Am Heart J 1937; 13: 633-646.

4. Lande A. Takayasu's arteritis and congenital coarctation of the descending thoracic and abdominal aorta: A critical review. Am J Roentgenol 1976; 127: 227-233.

5. Daimon S, Kitamura K. Coarctation of the abdominal aorta. Jpn Heart J 1964; 5: 557-562.

6. Connolly JE, Wilson SE, Lawrence PL, Fujitani. Middle aortic syndrome: distal thoracic and abdominal coarctation, a disorder with multiple etiologies. J Am Coll Surg 2002; 194: 774-781.

7. Uwabe K, Okada O, Harada M. Assending to descending aorta bypass for middle aortic syndrome. Circ J 2007; 71: 1162-1163.

8. Sen PK, Kinare SG, Enginner SD, Parulkar GB. The middle aortic syndrome. Br Heart J 1963; 25: 610-618.

9. Cohen JR, Birnbaum E. Coarctation of the abdominal aorta. J Vasc Surg 1988; 8: 160-164.

10. Vaccaro PS, Myers JC, Smead WL. Surgical correction of abdominal aortic coarctation and hypertension. J Vasc Surg 1986; 3: 643-648.

11. Gornik HL, Creaqer CM. Aortitis. Circulation 2008; 117: 3039-3051.

12. De Bakey ME, Garrett HE, Howell JF, Morris GC. Coarctation of the abdominal aorta with renal arterial stenosis: surgical considerations. Ann Surg 1967; 165: 830-843. 